\title{
INCONMENSURABILIDAD, INTRADUCIBILIDAD E INTENSIONALIDAD: UNA DISCUSIÓN ENTRE THOMAS KUHN Y DONALD DAVIDSON
}

\author{
INCOMMENSURABILITY, UNTRANSLATABILITY, AND \\ INTENSIONALITY: A DISCUSSION BETWEEN THOMAS KUHN \\ AND DONALD DAVIDSON
}

\section{Ángel Rivera-Novoa*}

\section{Resumen}

En este artículo se analizan dos de las estrategias de Kuhn para defender la inconmensurabilidad. Estas estrategias son la separación tajante entre interpretación y traducción y el reclamo de Kuhn de tener en cuenta los aspectos intensionales en los procesos de traducción. Se argumenta que ambas estrategias descansan en errores metodológicos. Por tanto, el argumento de Davidson contra la inconmensurabilidad puede defenderse aún.

Palabras clave: Inconmensurabilidad, intraducibilidad, intensionalidad, interpretación, Thomas Kuhn, Donald Davidson.

\begin{abstract}
In this paper, Kuhn's two argumentative strategies for defending incommensurability are analyzed. These strategies are, on one hand, the strong separation between interpretation and translation, and, on the other hand, the Kuhn's claim for taking into account the intensional aspects of translation. It is argued that both strategies rest on methodological mistakes in the process of radical interpretation. Hence, Davison's argument against incommensurability still could be defended.
\end{abstract}

Keywords: Incommensurability, Untranslatability, Intensionality, Interpretation, Thomas Kuhn, Donald Davidson.

Recibido: 2020-04-II Aceptado: 2020-06-22

* Universidad de San Buenaventura - Bogotá. E-mail: angelrivera32@gmail.com 
Tanto los defensores más fieles de las ideas de Thomas Kuhn como sus detractores más acérrimos están de acuerdo en que la introducción de la noción de "inconmensurabilidad" marcó un hito en la historia de la filosofía de la ciencia. Si bien antes de la publicación de La estructura de las revoluciones científicas (1962) se habían dado indicios importantes en torno a ciertas dificultades en la contrastación teórica, es sólo hasta el estudio de las revoluciones científicas de Kuhn que parece surgir un análisis sistemático al respecto. Sin duda, uno de los elementos importantes para que esto fuera posible es la perspectiva histórica de Kuhn. Ian Hacking describe las consecuencias de introducir la perspectiva histórica con las siguientes palabras:

Durante mucho tiempo los filósofos hicieron de la ciencia una momia. Cuando finalmente desenvolvieron el cadáver y vieron los restos de un proceso histórico de devenir y descubrimiento, crearon para sí la crisis de la racionalidad. Esto sucedió alrededor de 1960. [...] Fue una crisis porque sacudió nuestra antigua costumbre de pensar que el conocimiento científico es el pináculo de la razón humana. (Hacking 1983, p. 19)

En efecto, si se acepta que hay áreas de la empresa científica que son inconmensurables entre sí, pareciese entonces que tendríamos que redefinir el carácter 'racional' de la ciencia o, según algunos, abandonarlo, pues pareciera que los criterios clásicos de elección teórica serían incapaces de lograr su cometido.

La crisis de la racionalidad generada por la publicación de La estructura (1962) y de algunos otros escritos de Paul Feyerabend, desató un sin número de críticas que intentaban echar abajo las tesis que daban cabida a cualquier rasgo irracional en la empresa científica. En este artículo me interesa resaltar una de esas críticas: la de Donald Davidson (I974). Una de las virtudes de la crítica de Davidson fue la asociación entre la noción de esquema conceptual y la de intraducibilidad (o inconmensurabilidad). Habría esquemas conceptuales sólo en la medida en que hubiese intraducibilidad entre los lenguajes asociados a dos esquemas conceptuales.

I En sus artículos "Objetividad, juicios de valor y elección teórica" (1973) y "Racionalidad y elección de teorías" (1983b), Kuhn intenta mostrar que a pesar de que él rechaza que los criterios clásicos de selección teórica sean suficientes para explicar la racionalidad científica, él mismo no defiende tesis irracionalistas; más bien, la racionalidad de la elección simplemente atendería, según Kuhn, a otros criterios diferentes a los que clásicamente se habían postulado. No es el objetivo de este artículo examinar si Kuhn tiene o no implicaciones irracionalistas. Un interesante análisis que defiende la tesis de la inconmensurabilidad y simultáneamente la racionalidad en la elección teórica desde el pensamiento de Howard Sankey se encuentra en Melogno (20I4). 
La clave de la crítica de Davidson es mostrar, por medio de su método de la interpretación radical, que no hay posibilidad de intraducibilidad entre lenguajes (ni total ni parcial) y, así, se van al piso las condiciones de individuación de esquemas conceptuales. El modo en que Davidson plantea su crítica depende de una combinación de dos elementos importantes: el principio de caridad en conjunción con la noción de verdad tarskiana, la cual vincula conceptualmente las nociones de verdad y de traducción. El principio de caridad garantizaría que, en todo intento de interpretación de otro lenguaje, sea éste científico o no, el asumir que el otro está mayoritariamente de acuerdo con el intérprete es una condición de posibilidad de la interpretación misma. De esta manera, un defensor de la inconmensurabilidad debería, o bien aceptar que la interpretación entre lenguajes es imposible, lo cual parece problemático, o bien renunciar a que haya lenguajes inconmensurables entre sí. Por otra parte, el uso de la noción tarskiana de la verdad mostraría, según Davidson, que todo intento de interpretación, al pretender dar cuenta de las condiciones de verdad de los enunciados, necesariamente implicaría traducción. En esa medida al no haber lenguajes intraducibles o inconmensurables se tiene como resultado que "una forma de actividad que no pueda ser interpretada como lenguaje en nuestro lenguaje no es una conducta lingüística" (Davidson 1974, p. 185-I86)².

Algunos relativistas defendieron posteriormente un tipo de relativismo parcial justamente argumentando que los casos de intraducibilidad parcial eran posibles. Dos grandes líneas de argumentación, que tuvieron su origen en Thomas Kuhn, fueron tomadas por los relativistas: la separación entre interpretación y traducción y la exigencia de que la intensionalidad fuera dejada de lado en métodos de interpretación de corte davisoniano. Con ello, Thomas Kuhn y los relativistas parciales creen poder rescatar la idea de una intraducibilidad parcial. Mi objetivo en este texto es mostrar que ninguna de estas dos líneas obtiene el éxito que se propone.

\section{Interpretación y traducción}

Desde la "Introducción" de La estructura de las revoluciones científicas (1962), Kuhn ya anunciaba la inconmensurabilidad como la imposibilidad de acuerdo entre comunidades científicas en disputa. En particular,

2 A menos que en la bibliografía se indique lo contrario, las traducciones de las citas son mías. 
Kuhn habla de la inconmensurabilidad en términos de un desacuerdo en la forma de ver el mundo y de la manera de practicar la ciencia (cf. Kuhn I962, p. 25). Desde esta primera aparición del término, se revelan dos de los tres usos que adquiere el término "inconmensurabilidad" en el contexto de La estructura (I962), a saber, la inconmensurabilidad como desacuerdo irresoluble en las normas y problemas que debe asumir un paradigma (cf. Kuhn 1962, p. 230) y la inconmensurabilidad en la visión misma que se tiene del mundo, de tal modo que representantes de paradigmas diferentes habitan mundos diferentes (cf. Kuhn I962, p. I76).

Este tipo de inconmensurabilidad según el cual la visión del mundo o el mundo mismo resulta ser inconmensurable entre uno u otro paradigma fue en parte lo que generó una reacción crítica. Sin embargo, el tercer uso que se hace del término "inconmensurabilidad" en La estructura tiene que ver con la intraducibilidad que existe entre los lenguajes de las teorías en disputa (cf. Kuhn 1962, p. 23I). Es este uso del término el que Kuhn posteriormente defendió y profundizó. En la "Posdata" (I970) a La estructura, Kuhn limita el uso del término exclusivamente a su concepción semántica de tal suerte que la inconmensurabilidad se define exclusivamente en términos de intraducibilidad. Esta restricción del uso de la inconmensurabilidad será acompañada con un aspecto "local" de la inconmensurabilidad. En efecto, el modo en que Kuhn contesta a sus diferentes críticos es reduciendo la inconmensurabilidad a casos muy específicos de términos teóricos que se definen entre sí y esta representa su posición final (cf. Kuhn 1999, p. 34).

Para defender la intraducibilidad o inconmensurabilidad parcial, Kuhn, en su artículo "Conmensurabilidad, comparabilidad y comunicabilidad" (I983a) afirma que la crítica de Davidson a este tipo de inconmensurabilidad depende esencialmente de su método de interpretación radical, afirmando que si bien es cierto que una teoría $B$ interprete lo dicho por una teoría $A$, eso no implica que se elimine la posibilidad de una intraducibilidad parcial. El error de Davidson estriba en confundir, señala Kuhn, el proceso de interpretación con el proceso de traducción o, mejor, en comprometerse con la afirmación según la cual un proceso interpretativo implica necesariamente un proceso de traducción.

Kuhn intentará acudir a esta diferencia, pensando en que con esto puede escapar a las críticas en su contra que utilizan la noción o el método de 'interpretación'. Feyerabend acude a la misma estrategia. Según Feyerabend, las críticas que se han hecho hacia los relativistas suponen una premisa que no es clara: que el entendimiento de conceptos extraños supone necesariamente traducción (cf. Feyerabend 1987, p. 76). 
Barnes y Bloor (1982) creen que todos aquellos argumentos en contra del relativismo que acudan a alguna noción de traducción están errados. Según ellos, así como una lengua materna es aprendida sin necesidad de traducción, lo mismo puede ocurrir con otras lenguas. De esta forma, el entendimiento de una lengua y la noción de traducción están conceptualmente separados (cf. Barnes \& Bloor I982, pp. 39-40).

Si hay una separación entre interpretación (o entendimiento) y traducción, entonces puede ser el caso que haya una falla parcial de traducción - con lo que habría condiciones para individuar esquemas conceptuales-, sin que esto niegue la posibilidad de una comprensión de teorías inconmensurables. En lo que sigue, me centraré en la argumentación de Kuhn (1983a) y Sankey (I999), sólo con el objetivo de señalar el problema fundamental de esta aparente separación ${ }^{3}$.

Davidson, Putnam y Kitcher describen el resultado de una interpretación como una traducción, concluyendo así que el éxito de la traducción es incompatible con cualquier versión de la inconmensurabilidad, incluso la local. El argumento descansa en la dependencia, o no, que hay entre interpretación y traducción. Para Kuhn, interpretación y traducción son cosas muy diferentes. Kuhn afirma que la confusión es común, dado que los procesos de traducción incluyen a menudo un componente interpretativo.

La traducción es, según Kuhn, un proceso efectuado por una persona que sabe dos lenguajes. El traductor tiene un texto en un lenguaje y hace un texto equivalente en otro idioma, empresa que presupone dar cuenta de la igualdad de significados y de referencias de los términos y sentencias de los lenguajes involucrados ${ }^{4}$. En tal proceso, hay dos características importantes: por un lado, el lenguaje al que se traduce debe existir previamente al proceso de traducción mismo, es decir, el proceso de traducción debe dar cuenta del "hecho de que la traducción no ha

3 Lynch (1998), Hales (2006), Hacking (1982) y Carrier (200I) acuden igualmente a esta diferencia con el objetivo de defender su postura. Pero las diferencias argumentativas entre todos estos autores no son relevantes para los propósitos del presente artículo.

4 Esta noción de 'traducción' empleada por Kuhn hace pensar de inmediato en una noción pre-quineana de traducción. En efecto, para Quine, hay traducción incluso en el caso en el que el traductor sólo tiene dominio de un idioma (traducción radical). Además, la exigencia de Kuhn parece ser que el lenguaje traducido y el lenguaje al que se traduce, deben tener un conjunto de oraciones sinónimas y con exacta referencia de los términos y predicados involucrados. Sin embargo, para Davidson y para Quine, los procesos de traducción son posibles sin sinonimia y con inescrutabilidad de la referencia; además, la sinonimia es una noción oscura a la hora de dar cuenta de fenómenos como la interpretación. Sin embargo, mi crítica a este argumento depende de señalar un error metodológico anterior a los acá mencionados. 
cambiado los significados de las palabras o frases" (Kuhn I983a, p. 53). Esta característica es importante, pues, de no darse, en realidad no se estaría traduciendo de un lenguaje a otro, según Kuhn y Feyerabend, sino que lo que se haría sería una ampliación del vocabulario de alguno de los dos lenguajes - el lenguaje en donde se efectúa la traducción- (cf. Feyerabend 1987, p. 76). La otra característica importante puede ser descrita así: la traducción sólo consiste en palabras y oraciones que se reemplazan - no siempre una a una - por palabras y oraciones del lenguaje original. En otras palabras, las paráfrasis a las que acuden muchas veces ciertos traductores no hacen parte del proceso mismo de la traducción (cf. Kuhn I983a, p. 53).

Otra cosa sería la interpretación. Puede ser el caso que quien efectúe una interpretación sólo conozca uno de los dos lenguajes involucrados. En una situación de interpretación radical, el lingüista sólo tiene a su disposición una serie de ruidos ininteligibles. A través de las conductas de los hablantes y las condiciones del entorno, el lingüista plantea ciertas hipótesis para darle sentido a los enunciados del hablante. Así, si el intérprete tiene éxito, lo que ha hecho no sería más que aprender un nuevo lenguaje. De esta manera, tener éxito en la interpretación no implica necesariamente tener éxito en la traducción.

Según Kuhn, los ejemplos de Quine y Davidson confunden ambos procesos. Para interpretar la expresión 'gavagai' no es necesario encontrar un término correferencial o al menos similar en el propio lenguaje del intérprete. En vez de descubrir ese término, el lingüista puede aprender la expresión 'gavagai', del mismo modo en que se aprenden los términos del propio lenguaje como 'rojo', 'carro' o 'mamá' y esto no supone un proceso de traducción. Para estos casos, Kuhn reserva el término 'inconmensurabilidad', lo que daría como resultado una falla al menos parcial de la traducción, a pesar de que, en estos mismos casos, el proceso de interpretación sea exitoso (cf. Kuhn I983a, pp. 53-55).

Deseo argumentar que la distinción entre interpretar y traducir falla a la hora de ignorar ciertas cuestiones metodológicas del proceso de interpretación radical que se intenta atacar. En particular, quiero mostrar que este argumento, al ignorar lo que denominaré 'requisito de traducibilidad', no puede concluir, como lo pretende, que haya una separación tajante entre traducción e interpretación.

Lo primero que hay que advertir es que Davidson sí reconoce una diferencia entre traducción e interpretación. En su artículo "Radical Interpretation" (I973), Davidson señala que el uso del término "interpretación radical" en vez de "traducción radical" pone un énfasis sobre cier- 
tos aspectos semánticos (cf. Davidson I973, nota I) y, en "A Coherence Theory of Truth and Knowledge" (I983), Davidson dice que la diferencia más importante entre la traducción radical de Quine y su interpretación radical está en la elección de las causas relevantes para la interpretación o para la traducción: mientras Quine le da relevancia a la estimulación sensorial, Davidson sólo le da una importancia causal, pues, según él, las causas relevantes para la individuación del contenido son los acaecimientos y objetos del mundo mismo (cf. Davidson I983, p. I5I).

Ahora bien, aunque es cierto que hay una diferencia entre traducción e interpretación, sea cual sea, el análisis de Kuhn parece ignorar el hecho de que ambas nociones están estrechamente relacionadas, pues para que haya una interpretación correcta, se debe suponer a su vez la traducción, de tal modo que no sería inteligible hablar de la primera ignorando la última. Davidson impone como requisito de un método adecuado de interpretación lo siguiente:

[T]oda la teoría que se necesita [para un método de interpretación] es un método de traducción del lenguaje que se va a interpretar al lenguaje del intérprete. Tal teoría consistiría en el enunciado de un método efectivo para pasar de una oración arbitraria de la lengua ajena a una oración de un lenguaje familiar. (Davidson I973, p. I29)

Para la ejecución de una interpretación radical, se requiere de una teoría de la verdad tipo Tarski aplicada a lenguajes naturales. El proceso consiste en reunir la mayor cantidad de evidencia posible acerca de los asentimientos que un intérprete provoca en un hablante en situaciones muy particulares —esto es, en la emisión de oraciones ocasionales- - A partir de la evidencia reunida, se formulan hipótesis y axiomas (de base y recursivos) que finalmente generan oraciones como la siguiente: " $E s$ regnet’ es verdadera en alemán si y sólo si está lloviendo”, de forma tal que la oración en cuestión resulta verdadera.

¿Qué sucede si la teoría entraña oraciones del tipo "La nieve es blanca' es verdadera en L si y sólo si el pasto es verde”? Tal oración es verdadera, pero no interpretativa. La equivalencia material de los teoremas, esto es, el hecho de que las V-oraciones de la teoría sean verdaderas, no es una condición suficiente para que una interpretación sea exitosa. En el esquema “'o' es verdadera en L si y sólo si p", si el lenguaje-objeto está contenido en el metalenguaje, $p$ sería la oración designada por $o$; si no, se debe realizar una traducción de la oración en el metalenguaje. De este modo, a partir de los axiomas, además de la evidencia reunida, las V-oraciones derivadas no sólo serán equivalentes 
materialmente, sino que, para que haya una interpretación correcta, $p$ debe ser una traducción de $o$. No es suficiente la equivalencia material, sino que también es necesario lo que quiero denominar "requisito de traducibilidad". Sólo por medio de tal requisito podemos, por un lado, evitar la derivación de V-oraciones anómalas y, por otro, hacer que una teoría de la verdad funcione como una teoría de la interpretación.

Se puede objetar que si Davidson de hecho utiliza la noción de 'traducción' para dar cuenta de un proceso de interpretación, entonces estaría cayendo en una petición de principio. Al ser la 'traducción' una noción intensional, se estaría presuponiendo aquello sobre lo que se quiere dar luz, a saber, el significado de las emisiones de un hablante. No obstante, creo que el análisis de Davidson conduce a decir que interpretación implica traducción, aun sin presuponer, de entrada, la traducción, lo cual generaría la petición de principio.

Davidson invierte el análisis tarskiano acerca de la verdad, pues mientras Tarski parte de la noción de 'traducción' (i.e., parte del significado) para dar luces sobre el concepto de verdad, Davidson toma la noción de 'verdad' como básica para arrojar luz acerca de la naturaleza del significado. Dice Davidson:

Dado que Tarski estaba interesado en definir la verdad [...] podía dar por sentado el concepto de traducción. Pero en la interpretación radical, esto es precisamente lo que no puede ser asumido. Así, en vez de esto, he propuesto algunos constreñimientos empíricos para la aceptación de una teoría de la verdad que pueda ser establecida sin apelar a conceptos tales como significado, traducción o sinonimia, aunque no sin un cierto entendimiento de la noción de verdad. Mediante cierta forma de razonamiento, he intentado mostrar que si los constreñimientos son satisfechos por una teoría, entonces las V-oraciones que surgen de la teoría de hecho tendrán traducciones de s reemplazando a 'p'. (Davidson I976, pp. I72-I73, énfasis añadido).

De esta manera, incluso cuando no supongamos de entrada la noción de traducción, el 'requisito de traducibilidad' aún se debe mantener si queremos una interpretación adecuada, de tal modo que interpretación en efecto implica traducción si se cumplen los constreñimientos empíricos de la teoría'. Así pues, no es posible separar la noción de 'ver-

5 Entre los constreñimientos empíricos, Davidson señala algunos tales como la legaliformidad de las V-oraciones que les permitirían resistir contra-fácticos (cf. Davidson 1976) o la estructura composicional de los lenguajes (cf. Davidson I967) así como las mismas condiciones de verdad que posibilitan las diversas interpretaciones (cf. Davidson 1973). Todos estos constreñimientos deben ser verificables empíricamente y, si lo son, entonces la interpretación implica traducción. Para ver tres análisis y réplicas a estos 
dad' de la noción de 'traducción' y, como el método de interpretación radical, para que sea considerado exitoso, necesita de la noción tarskiana de la verdad, es imposible concebir una interpretación sin acudir a la noción de traducción. La defensa de Kuhn descansa entonces en un error metodológico, ya que es a través del método de interpretación radical que Davidson critica la posibilidad de una intraducibilidad parcial. La defensa resultaría entonces una defensa ad hoc, pues no se ataca el principio mismo, sino que se declara y se da por sentada la separación conceptual entre traducir e interpretar.

En su artículo "Incommensurability, Translation and Understanding" (199I), Sankey quiere defender la idea de que la falla parcial de traducción no es incompatible con el entendimiento o la comprensión de lo que él denomina "sub-lenguajes" inconmensurables (cf. Sankey I99I, p. 4I6), para de esta manera poder reforzar la intuición de Kuhn según la cual hay una separación entre los procesos de traducción y los procesos de interpretación. Para culminar esta sección, revisaré la estrategia de Sankey con el fin de resaltar algunos puntos problemáticos de la misma.

Según Sankey, una condición para que haya traducción es que haya condiciones semánticas tales que el lenguaje al que se traduce tenga oraciones con el "mismo significado" del lenguaje traducido (cf. Sankey I99I, pp. 4I6-4I7). Además, la inconmensurabilidad no niega, dice el autor, la posibilidad de una traducción aproximada, sino la posibilidad de una traducción exacta. Ante esto vale la pena resaltar dos cosas: por un lado, Quine muestra que esta noción de "mismidad de significado" es oscura e irrelevante para las cuestiones de traducción radical como lo señalé en una nota al pie anterior. Por otro lado, si la inconmensurabilidad propuesta admite que haya traducciones aproximadas, aunque no exactas, entonces sería una noción de inconmensurabilidad superflua que no sería más que la propia indeterminación que Quine y Davidson aceptan. No obstante, mis objeciones más centrales son de otra índole.

Teniendo en cuenta esta noción de traducción, Sankey pasa inmediatamente a hacer la separación entre traducción y entendimiento. $\mathrm{Al}$ igual que Kuhn (1983a) y Barnes \& Bloor (1982), Sankey acude al hecho de que el aprendizaje de la primera lengua no requiere de la tarea de equiparar significados iguales. Del mismo modo, un lingüista podría entender el lenguaje de una cultura extraña sin acudir a métodos de traducción, o un científico podría entender el lenguaje de una teoría rival sin tener que traducirlo (cf. Sankey I99I, pp. 4I7-4I8). 
Ahora bien, se podría decir que no es lo mismo el aprendizaje de la primera lengua al aprendizaje de una segunda lengua. Así, aunque en el aprendizaje de la primera lengua no es necesaria la traducción, puede que en el de la segunda sí lo sea (cf. Achinstein 1968, p. 97). Pues bien, Sankey acepta que el aprendizaje de una y otra cosa son diferentes, pero señala que la inconmensurabilidad se da entre sub-lenguajes teóricos intraducibles que pueden pertenecer a un mismo lenguaje natural y no entre lenguajes naturales (cf. Sankey 1991, p. 419).

Pero este punto es problemático y parece surgir una paradoja: si existen dos sub-lenguajes, es obvio que son sub-lenguajes de un mismo lenguaje natural. Así, las oraciones que pertenecen a ambos sub-lenguajes, hacen parte de un mismo lenguaje. Pero si esto es así, ¿en qué sentido podemos decir que hay fallas de traducción entre oraciones que pertenecen a un mismo lenguaje? El problema con la propuesta de Sankey estriba en que se llega a una paradoja: para que haya intraducibilidad se requieren al menos dos lenguajes, pero la intraducibilidad propuesta por Sankey es una intraducibilidad entre oraciones de un mismo lenguaje, lo cual resulta contradictorio.

Se podría afirmar que esta respuesta es demasiado simplista y que es adjudicarle a Sankey una visión muy ingenua de la idea que en realidad está detrás. No importa que los sub-lenguajes hagan parte de un solo lenguaje natural más amplio. El punto es que, en definitiva, los recursos semánticos del lenguaje de una teoría Ti son insuficientes para expresar las proposiciones de una teoría T2 y viceversa. Es en ese sentido en el que son intraducibles - aunque ambos lenguajes pertenezcan, por ejemplo, al español-. El mismo Kuhn parece estar pensando en ello cuando intenta limitar el sentido del término "inconmensurabilidad": "alguna proposición que puede ser verdadera (o falsa) en un lenguaje podría incluso no ser formulada en otro" (Kuhn I999, p. 35). Si bien en este pasaje no se hace alusión a alguna clase de "sublenguajes" como los que propone Sankey, sí se muestra que lo que parece ser fundamental en la tesis de la inconmensurabilidad es la insuficiencia de los recursos semánticos de un lenguaje para dar cuenta de los significados de otro lenguaje.

A pesar de lo anterior, se puede seguir sosteniendo que no tiene sentido pensar en intraducibilidad de sub-lenguajes. Sea que un sub-lenguaje I no capture en principio lo dicho por un sub-lenguaje 2, al compartir ambos un lenguaje natural, sería legítimo simplemente ampliar el vocabulario y los recursos semánticos de i para poder introducir proposiciones de 2. Así, como es posible ampliar los sub-lenguajes, no hay posibilidades de intraducibilidad. Otra opción es utilizar una pará- 
frasis en el sub-lenguaje para dar cuenta de lo dicho en el otro sub-lenguaje. Un caso de ampliación de vocabulario se da cuando se introduce un nuevo término para capturar un concepto que antes no se podía capturar. La paráfrasis, en cambio, no introduce un nuevo término, sino que presenta una descripción del concepto de la otra teoría.

Por supuesto, Kuhn, Feyerabend y Sankey argumentarían, como ya lo he señalado, que ampliar un lenguaje, o utilizar paráfrasis, no es lo mismo que traducir y que ambos recursos en realidad son un indicio de la imposibilidad de traducción. Ante esto, sólo puedo decir que es cierto sólo si se acepta una premisa que estos autores comparten - a la cual también ya me referí-, a saber, que los lenguajes, si amplían su vocabulario, dejan de ser lo que eran y pasan a ser un lenguaje diferente (cf. Kuhn I983a, p. 52); por tanto, ampliar un lenguaje no es garantía de traducción. Ésa es, sin embargo, una visión muy estática de los lenguajes (tal visión implica que los lenguajes nunca pueden cambiar). Por el contrario, los lenguajes son cambiantes: ampliamos constantemente nuestro vocabulario y en algunas cosas excluimos palabras obsoletas. Las palabras mismas son cambiantes. En palabras de Wittgenstein:

[P]regúntate si nuestro lenguaje es completo - si lo era antes de incorporarle el simbolismo químico y la notación infinitesimal, pues éstos son, por así decirlo, suburbios de nuestro lenguaje. (¿Y con cuántas casas o calles comienza una ciudad a ser ciudad?). Nuestro lenguaje puede verse como una vieja ciudad: una maraña de callejas y plazas, de viejas y nuevas casas, y de casas con anexos de diversos períodos; y esto rodeado de un conjunto de barrios nuevos con calles rectas y regulares y con casas uniformes. (Wittgenstein 1953, \$18).

El argumento para salvar la separación radical entre interpretación y traducción dependería, por tanto, de asumir una noción de lenguaje estática que parece no corresponderse con nuestra imagen intuitiva de lo que constituye un lenguaje, a saber, que es algo esencialmente mutable.

Se podría insistir en lo siguiente: sea que los lenguajes sean cambiantes o no, al ampliar el vocabulario definitivamente no hay traducción. Pues bien, puedo estar de acuerdo en que es bastante dudoso que ampliar el vocabulario (o utilizar paráfrasis) sea un proceso de traducción, pero al efectuar estos procesos, eliminamos la posibilidad de intraducibilidad. Esto último es, no obstante lo más importante. Si se elimina la posibilidad de intraducibilidad (sea traduciendo, ampliando el lenguaje o por paráfrasis), se pierden las condiciones de individuación de esquemas conceptuales y, con ello, la posibilidad misma de la inconmensurabilidad. 
Una segunda crítica estudiada por Sankey es aquella que depende del 'principio de efabilidad'. Según tal principio, todo lo que puede ser pensado es expresable en cualquier lenguaje ${ }^{6}$. Sean LI y L2 dos lenguajes intraducibles entre sí. Si el principio de efabilidad es cierto, entonces cualquier cosa que sea pensada por un hablante de L2, puede ser dicha en Li. Pero si eso es así, no hay una intraducibilidad entre ambos lenguajes.

Sankey afirma que el principio contrario no es falso de una manera obvia. Un lenguaje puede carecer de los recursos semánticos suficientes para expresar algún pensamiento. El principio de efabilidad tendría que ser debilitado así: cualquier cosa que pueda ser pensada, debe poderse expresar en algún lenguaje (Sankey 199I, pp. 420-42I). Sin embargo, tal argumento presenta el siguiente problema: no se ha argumentado que los lenguajes carezcan de los recursos semánticos suficientes para decir lo que puede ser pensado. Para argumentar esto, sin embargo, sería necesario romper la estrecha relación que hay entre creencia y significado y, así mismo, la relación que hay entre interpretación de oraciones y atribución de creencias. No obstante, en un proceso de interpretación radical, la evidencia primaria de interpretación, esto es, el hecho de que el hablante sostenga como verdaderas ciertas oraciones, es evidencia justamente para decir que la mencionada relación existe: el hablante cree lo que dice - aunque, en principio, el intérprete no sepa qué creencia tiene el hablante, ni cuál es el significado de su emisión-.

Un último intento para mostrar que es posible la intraducibilidad entre sub-lenguajes teóricos la brinda el propio Kuhn. Kuhn comienza su argumentación en este punto analizando los dos siguientes supuestos de la crítica de Davidson:

Davidson reconoce, como yo, que el traductor radical de Quine es de hecho un aprendiz del lenguaje y que lo que él ha aprendido no puede integramente ser traducido en el lenguaje que él trae desde casa. Pero Davidson supone, y yo no, que una vez se han comprendido los términos del lenguaje recientemente adquirido, el aprendiz del lenguaje puede enriquecer su lenguaje nativo añadiendo las palabras faltantes. El enri-

6 Un principio similar es postulado por John Searle en Actos de habla (2009). Searle lo denomina 'principio de expresabilidad' y lo define de la siguiente manera: para todo lo que un hablante quiera decir existe una expresión en el lenguaje para decirlo (cf. Searle 2009, p. 29). Un hablante puede, o bien carecer de recursos efectivos para expresar lo que quiere o bien desconocer el vocabulario que tiene a su disposición. De acuerdo con Searle, lo primero se soluciona ampliando el vocabulario del lenguaje y lo segundo con una ampliación del conocimiento de los recursos lingüísticos disponibles. 
quecimiento habría entonces eliminado la inconmensurabilidad. (Kuhn I999, p. 35).

Lo primero que vale la pena resaltar es que estos dos supuestos que se atribuyen a Davidson parecen ser explícitamente rechazados por él. Parte de las razones ya han sido expuestas: el requisito de traducibilidad presente en la interpretación radical de Davidson así lo muestra. Una falla en la traducción significaría para Davidson una evidencia de que lo que se intentaba traducir no era en realidad una conducta lingüística. Quizás Kuhn está pensando en los casos de indeterminación quineana. Pero estos casos, aceptados por Davidson, no constituyen ellos mismos casos de intraducibilidad. Sin embargo, lo que me interesa es la última parte del argumento de Kuhn. Según él, cuando se intenta ampliar o enriquecer el vocabulario del metalenguaje en el que se realiza la interpretación o la traducción, habría un conjunto de proposiciones que no pueden ser sostenidas consistentemente: "enriquecer el vocabulario conceptual newtoniano con términos aristotélicos (o viceversa) causaría contradicciones acerca de fenómenos observables en el lenguaje mismo. Los usuarios de un lenguaje que contuviera tales contradicciones no tratarían con la naturaleza exitosamente" (Kuhn I999, p. 36).

En este punto, sin embargo, Kuhn parece tener un supuesto adicional que no es del todo claro. El supuesto consiste en afirmar que si enriquezco el vocabulario para traducir o dar cuenta de un sublenguaje alternativo, entonces el metalenguaje debe comprometerse con las verdades expresadas en ese otro lenguaje y, sólo así, es que surgiría la contradicción. Sin embargo, la convención tarskiana implica que lo que debe asumirse como verdadera es la V-oración y no sus partes individuales. En efecto, en la V-oración “ “o” es verdad en L si y sólo si p” podemos suponer que la oración " $p$ " tiene términos nuevos con los cuales se efectúa la traducción. El bicondicional entero puede ser verdadero, pero eso no quiere decir que el usuario del lenguaje en el que se traduzca la oración deba comprometerse con la verdad de la oración "o" ni con su correspondiente traducción " $p$ ”. Kuhn debió recordar que un bicondicional puede ser verdadero en el caso en que sus proposiciones componentes sean ambas falsas.

\section{Intensionalidad e inconmensurabilidad}

En esta segunda y más breve sección, analizaré otro tipo de argumentación de Kuhn, según el cual, los métodos davidsonianos, al ser extensio- 
nales, dejan de lado la intensionalidad, donde justamente tiene campo la intraducibilidad al menos parcial y, por tanto, la inconmensurabilidad entre teorías?.

Los métodos de traducción, según Kuhn, no pueden verse sólo desde el punto de vista referencial de los términos, sino además desde un punto de vista intensional o semántico, que es, en últimas, lo que conduce a la inconmensurabilidad. Un intérprete radical puede relacionar términos coextensivos entre la lengua del hablante y la propia; sin embargo, señala Kuhn, debido a los diversos contextos interpretativos, la interpretación no daría cuenta de la intensionalidad de los términos (cf. Kuhn I983a, p. 64). El error en la crítica a la inconmensurabilidad consistiría en buscar algo como lo siguiente: "una teoría de la traducción basada en una semántica extensional y, por tanto, restringida a la preservación del valor veritativo o a la equivalencia como criterio de adecuación" (Kuhn I983a, p. 65). Si la mirada se posa exclusivamente sobre el aspecto extensional del lenguaje, la inconmensurabilidad, en efecto, parece no tener lugar. El problema es que un método de interpretación o traducción no puede dejar de lado los significados ni las intensiones, pues su objetivo central será capturarlas. Una vez el traductor enfoca la mirada en las intensiones y los significados el fenómeno de la intraducibilidad se hace patente.

De esta manera, se cree entonces salvar una clase de inconmensurabilidad local criticando el hecho de que filósofos como Quine o Davidson hablen de una teoría de la traducción —o interpretación - restringida exclusivamente a un plano extensional, dejando al margen el intensional, que es el relevante para la inconmensurabilidad. Los fenómenos de intraducibilidad no se darían en un plano extensional, pero sí en uno intensional.

En "Truth and Meaning" (1967) y en "Radical Interpretation" (I973), Davidson defiende la relación entre la interpretación radical con una concepción holista del significado en donde el significado de una oración depende del significado del conjunto de oraciones de un lenguaje. Este holismo, cree Davidson, cumple con la condición de derivar todas las oraciones de la forma "o significa que $p$ ". Surge, sin embargo, una dificultad con este tipo de oraciones, a saber, el uso de la expresión "significa que $p$ " abre la puerta a los problemas que trae consigo la intensionalidad, esto es, al haber contextos opacos referencialmente, la substitución de términos coextensivos podría no conservar el valor de verdad

$7 \quad$ Este tipo de argumentación puede también encontrarse en Sankey (1994), Hales (1997) y en Carrier (200I). 
de las oraciones. Por tal motivo, Davidson reemplaza este esquema de oración introduciendo la estructura de V-oraciones, las cuales por definición operan en un plano estrictamente extensional. La relación entre la teoría de la verdad de Tarski y el concepto de significado radica en que la definición ofrece las condiciones suficientes y necesarias para la verdad de toda oración; pero dar tales condiciones es precisamente dar el significado de una oración.

Además, si la interpretación radical es un método para determinar el significado de las emisiones de los hablantes, siendo el significado una noción intensional, una teoría adecuada que pretenda dar cuenta del significado no puede presuponer nociones intensionales, pues caería en una petición de principio. Davidson señala esto en varios pasajes:

Lo que exigimos de una teoría del significado para un lenguaje $L$ es que sin apelar a ninguna noción semántica (adicional) coloque restricciones suficientes sobre el predicado 'es V' para entrañar todas las oraciones obtenidas a partir del esquema (V) cuando 'o' se reemplaza por una descripción estructural de una oración de $L$ y 'p' por esa oración. (Davidson 1967, p. 23, énfasis añadido).

Así, al formular una teoría del significado se debe evitar presuponer nociones intensionales para no caer en una petición de principio. En "Radical Interpretation", Davidson afirma:

En la interpretación radical, sin embargo, se supone que la teoría proporciona una comprensión de emisiones particulares que no se da de antemano, de modo que la última evidencia para la teoría no puede consistir en interpretaciones correctas de muestras. Para habérselas con el caso general, la evidencia tiene que ser de una clase que tendría que estar disponible a cualquiera que no sepa previamente cómo interpretar las emisiones para tratar con las cuales está diseñada la teoría: tiene que ser evidencia que pueda ser enunciada sin uso esencial de conceptos lingüísticos tales como significado, interpretación, sinonimia y cosas parecidas. (Davidson I973, p. I28, énfasis añadido).

Otra razón por la cual es erróneo presuponer en la interpretación radical nociones intensionales es que, dado que la interpretación radical es un método con pretensiones de verificación empírica, la evidencia aludida no debe ser enunciada por medio de ninguna noción intensional, pues, de tal modo, se presupondría en la evidencia aquello de lo cual se quiere dar cuenta ${ }^{8}$.

8 Algo adicional, que da cuenta de lo anterior, es lo dicho por Davidson en una nota al pie agregada en 1982 a "Truth and Meaning" (I967) y en "Reply to Foster" (1976). 
Es entonces un error metodológico acusar a Davidson por el hecho de construir una teoría del significado puramente extensional, ya que al involucrar nociones como las de 'significa que', 'sinonimia', 'significado', etc., se presupondría, en diversos puntos, aquello sobre lo que se quiere dar luz. Por otra parte, no sólo los referentes de los términos y las oraciones de un hablante son capturados por la teoría de la traducción de Davidson. Gracias a la teoría causal del contenido en conjunción con el holismo semántico se puede determinar el significado de los términos y las oraciones en un proceso de interpretación radical, sin que la teoría deje de ser extensional. Por tanto, si acudiendo a una interpretación radical, que tiene una naturaleza extensional, garantizamos la traducción enre lenguajes, entonces la inconmensurabildiad no tendrá lugar, incluso cuando la metodología interpretativa no sea intensional ${ }^{9}$.

\section{Conclusiones}

He expuesto las dos principales líneas argumentativas para rescatar la intraducibilidad parcial planteadas por Kuhn y he mostrado que ambas caen en errores metodológicos. No podemos separar interpretación de traducción, porque ello supone ignorar el requisito de traducibilidad de la metodología davidsoniana de la interpretación radical. Por otra parte, los métodos de interpretación no deben ser formulados en un lenguaje intensional que le dé un supuesto campo a la intraducibilidad parcial, ya que se caería en una petición de principio. Esto no supone que del hecho de que los métodos de interpretación sean extensionales se siga que no se dé cuenta del significado.

Creo que la estrategia más adecuada para poder salvar una noción de inconmensurabilidad es tratar de hacerlo sin apelar a los fenómenos de intraducibilidad. En últimas, a Kuhn le interesa garantizar que I) existan conceptos teóricos que están íntimamente relacionados entre sí y que 2) debido a la existencia de dichos conceptos no sea posible decidir neutralmente entre redes de conceptos que sean rivales. Esto además garantizaría un elemento que es muy importante en los últimos escritos

Allí, Davidson introduce la idea de las V-oraciones deben tener una estructura legaliforme capaz de resistir contra-fácticos ( $c f$. Davidson 1967, nota II). Ahora bien, si las V-oraciones son leyes, entonces tales oraciones dan cuenta de lo intensional.

9 Un debate interesante, pero que desborda los propósitos del presente artículo, tiene que ver con si en efecto una teoría extensional del significado es enteramente interpretativa. Para ver una defensa de las teorías extensionales del significado tipo Davidson frente a críticas intensionalistas, cf. Rivera-Novoa \& González (20I7). 
de Kuhn, a saber, la existencia de taxonomías teóricas que no se pueden trasponer. Si es posible defender la existencia de estas redes de conceptos y taxonomías sin acudir a la intraducibilidad (ni tampoco a la intensionalidad), tendríamos una noción de inconmensurabilidad que escaparía a la crítica de Davidson y que, por tanto, no se comprometería con el espinoso asunto de la intraducibilidad ni con la problemática existencia de esquemas conceptuales. Ésa es una alternativa que valdría la pena considerar ${ }^{10}$.

\section{Referencias bibliográficas}

Achinstein, P. (1968). Concepts of Science. Baltimore: Johns Hopkins university Press.

Barnes, B. \& Bloor, D. (1982). "Relativism, Rationalism and Sociology of Knowledge”. En Hollis \& Lukes (eds.), Rationality and Relativism, 2I-43. MIT Press,.

Carrier, M. (200I). "Changing Laws and Shifting Concepts". En Hoyningen-Huene, P. \& Sankey, H. (eds.), Incommensurability and Related Matters, 64-90. Kluwer Academic Publishers.

Davidson, D. (1967). "Truth and Meaning". En Davidson (2001a), 17-36. (I973). "Radical Interpretation". En Davidson (2001a), I25-I39. (I974). "On the Very Idea of Conceptual Scheme". En Davidson (200Ia), I83-198. (I976). "Reply to Foster". En Davidson (200Ia), I7I-I79. (I983). "A Coherence Theory of Truth and Knowledge". En

Davidson (200Ib), I37-I57.

(200Ia). Inquires into Truth and Interpretation. Oxford: Oxford University Press. (200Ib). Subjective, Intersubjective, Objective. Oxford: Oxford University Press.

Feyerabend, P. (1987). "Putnam on Incommensurability". British Journal of Philosophy of Science, 38: 75-92.

Fodor, J. A. \& LePore, E. (1992) Holism:A shopper's Guide. Oxford: Blackwell.

Io Un intento de esta posibilidad se expone en Rivera-Novoa (2020), donde se intenta articular una clase de relativismo parcial que no se comprometa con la existencia de esquemas conceptuales. La inconmensurabilidad sin intraducibilidad tendría lugar cuando dos lenguajes sólo se pueden traducir por paráfrasis o por ampliación del vocabulario. 
Hacking, I. (1982). "Language, Truth and History". En Hollis, M. \& S. Lukes (eds.). Rationality and Relativism, 47-66. Cambridge, Mass.: The MIT Press. (1983). Representar e intervenir. Traducción de S. F. Martínez, México: Paidós, 200I.

Hales, S. (1997). "A Consistent Relativism". Mind I06(42I): 32-52. (2006). Relativism and the Foundations of Philosophy. Cambridge, Mass.: The MIT Press.

Kuhn, T. (1998 [1962]). La estructura de las revoluciones científicas. Traducción de A. Contín. México: Fondo de Cultura Económica. (1970). "Posdata". En Kuhn (1998 [1962]): 268-319. (I973). "Objectivity, Value Judgment, and Theory Choice". En Kuhn (1977), 344- 364. (I977). La tension esencial. Traducción de R. Helier. México: Fondo de cultura Económico, 1987. (I983a). "Conmensurabilidad,comparabilidadycomunicabiliad". En Kuhn (2000), 47-75. (1983b). "Racionalidad y elección de teorías". En Kuhn (2000), $247-255$. (I999). "Remarks on incommensurability and translation". En R. Favretti, G. Sandri, and R. Scazzieri (eds.) Incommensurability and Translation: Kuhnian Perspectives on Scientific Communication and Theory Change, 33-37. Cheltenham and Northampton: Edward Elgar. (2002 [2000]). El camino desde la estructura. Traducción de A. Beltrán y J. Romo. Barcelona: Paidós.

Lepore, E. \& Loewer, B. (1989). "What Davidson Should Have Said". En Brand, J. \& Gombocz, W. (eds.), The Mind of Donald Davidson, I49I56. Amsterdam-Atlanta: Rodopi.

Ludwig, K. (1999). "Theories of Meaning, Truth and Interpretation". En Zeglen, U.M. (ed.) Donald Davidson. Truth, Meaning and Knowledge. 27-46. London: Routledge,

Lynch, M. (1998). Truth in Context: An Essay on Pluralism and Objectivity. Cambridge: The MIT Press.

Melogno, P. (20I4). "Racionalidad y elección de teorías: una aproximación a Howard Sankey". Revista colombiana de filosofía de la ciencia I4(29): II3-I38.

Rivera-Novoa, Á., \& González, Ó. L. (2017). "Davidson y el dilema intensional-extensional: aporías de la interpretación. Discusiones Filosóficas I8(30): 79-95. 
Rivera-Novoa, Á. (2020). "Relativismo sin esquemas conceptuales". Ideas $y$ valores 69(I73): 77-IO2.

Sankey, H.(199I). "Incommensurability, Translation and Understanding”. En The Philosophical Quarterly 4I, I65: 4I4-426. (1994). The Incommensurability Thesis. Aldershot: Ashgate.

Searle, J. (2009). Actos de habla. Traducción de D. Lara. Madrid: Cátedra. Wittgenstein, L. (1986 [1953]) Investigaciones filosóficas. Traducción de A. García Suárez y U. Moulines. México: Universidad Autónoma de México. 